\title{
Preferência de Gestantes pelo Parto Normal ou Cesariano
}

\author{
Lígia Ebner Melchiori \\ Ana Cláudia Bortolozzi Maia \\ Rita Nathalia Bredariolli \\ Raquel Ishikawa Hory \\ Universidade Estadual de São Paulo
}

\begin{abstract}
RESUMO
O estudo investigou a preferência pelo tipo de parto em 40 gestantes primigestas por meio de uma entrevista semiestruturada. A maioria das gestantes (75\%) relatou preferir o parto normal, principalmente porque acreditam ter uma rápida recuperação e ser melhor para elas e/ou bebês. $15 \%$ das gestantes preferiram a cirurgia cesariana porque consideram ser um parto sem dor e essas preferências, em geral, foram influenciadas por amigas. A maioria das gestantes afirmou que diante do parto temem: sentir dor/sofrer, acontecer algo com elas e/ou bebês, a situação desconhecida ou avaliada como negativa e a presença de pessoas de confiança, como o marido, a mãe ou o médico, poderia minimizar esse temor. Esses resultados reforçam as contribuições de profissionais da saúde, como os psicólogos, no atendimento das necessidades psicossociais de gestantes diante do parto e do nascimento.
\end{abstract}

Palavras-chave: parto normal; cesárea; gravidez; psicologia da saúde.

\begin{abstract}
Pregnant Women's Preference for Vaginal or Caesarean Childbirth

The study investigated the preference for delivery in 40 pregnant women using a semi-structured interview. Most women (75\%) reported a preference for vaginal delivery, mainly because they believe it will result in a speedy recovery and be better for them and/or their babies. Of the women interviewed, $15 \%$ preferred a caesarean section because they considered it be less painful. These preferences, in general, were influenced by friends. Most women said they had fear of parturition due to: feelings of pain/suffering, concern of something happening to them or their babies and because the situation is unknown or assessed in a negative fashion. The presence of trusted people, like one's husband, mother or doctor, could minimize this fear. These results reinforce the contributions of health professionals, such as psychologists, in answering the psychosocial needs of pregnant women in situations of delivery and birth.
\end{abstract}

Keywords: vaginal birth; caesarean section; pregnancy; health psychology.

Em países ocidentais, desde a década de setenta, há registros de aumento das taxas de cesariana (Halvorsen, Nerum, Oian \& Sorlie, 2008) que tem preocupado muitos pesquisadores de diferentes áreas da saúde médica, psicológica e social. Várias pesquisas têm chamado a atenção para o elevado índice de cesarianas realizadas no país (Barbosa \& cols., 2003; Carniel, Zanolli \& Morcillo, 2007; Dias \& Deslandes, 2004; Diniz \& Duarte, 2004; Faisal-Cury \& Menezes, 2006; Faúndes \& Perpétuo, 2002; Hopkins, 2000; Miranda, 2003; Silveira \& Santos, 2004; Souza, 2004). Este índice é maior à medida que aumenta o nível socioeconômico da mulher, independentemente do risco obstétrico (Sociedade Civil de Bem-Estar Familiar no Brasil, 1997; Yazlle \& cols., 2001).

Hopkins (2000) afirma que a taxa de cesariana nos hospitais privados brasileiros está acima de $70 \%$ e, algumas vezes, excede $90 \%$ dos partos, embora a Organização Mundial de Saúde (OMS) recomende que apenas $15 \%$ dos partos ocorram por meio de partos cirúrgicos, isto é, que a cesariana seja uma indicação apenas quando há necessidade de garantir a saúde da mãe e do feto. 
O Ministério da Saúde no Brasil tem incentivado o parto normal, por meio de campanhas, programas e portarias, por defender que este tipo de parto oferece menor risco de infecções e complicações maternas dentre outras vantagens (Gama, 2007; Hotimsky, Rattner, Venancio, Bógus \& Miranda, 2002; Oliveira, Riesco, Miya \& Vidotto, 2002; Serruya, Lago \& Cecatti, 2004) e, no entanto, os índices de cesáreas no Brasil mantêm-se ainda elevados (Carniel \& cols., 2007; Silveira \& Santos, 2004). Diante deste quadro, o Ministério da Saúde e a Agência Nacional de Saúde Suplementar continua promovendo campanhas sobre o parto normal, incentivando a sua prática em serviços públicos e privados ("Novas ações", 2009).

Muitos autores compartilham preocupações pelos altos índices de parto cesariano no país por diversos motivos: a) exposição desnecessária aos riscos de submissão a procedimento cirúrgico (Perpétuo, Bessa \& Fonseca, 1998; Quadros, 2000); b) aumento dos gastos com serviços de saúde, do ponto de vista hospitalar e/ou do médico (Fonseca, 1998; Perpétuo \& cols., 1998; Souza, 1994); c) aumento da mortalidade materna e fetal (Faúndes \& Cecatti, 1991; Souza, 1994); d) maior probabilidade de nascimento de bebês prematuros, de baixo peso e com transtornos respiratórios e/ou neurológicos (Souza, 1994).

A prática do parto cesariano tem sido recorrente, especialmente entre as clínicas particulares, e nem sempre se trata de uma opção que atende às reais necessidades psicossociais das gestantes, mas favorece interesses diversos dos profissionais envolvidos. Por exemplo, ao determinar a cirurgia cesariana, o(a) médico(a) pode manejar o tempo de duração do parto e o horário de realização, auferir maiores ganhos financeiros, associar o procedimento cirúrgico de cesariana com a execução de ligação tubária e ainda esconder a falta de preparo na condução de partos normais (Diniz, 2005; Hotimsky \& cols., 2002; Martins-Costa \& Ramos, 2005; Oliveira \& cols., 2002; Perpétuo \& cols., 1998; Potter \& cols., 2001). Silveira e Santos (2004) comentam que há indicações relacionadas ao sofrimento fetal e riscos à mãe que justificam a cesárea, porém, muitas vezes há uma avaliação subjetiva não relacionada às questões clínicas, que impõe a comodidade do médico sobre a necessidade da mãe.

Oliveira e cols. (2002) relatam que, entre os médicos, as indicações mais frequentes para a realização da cesárea foram: ocorrência de cesárea anterior, sofrimento fetal e apresentação pélvica. No entanto, em $47,5 \%$ dos casos, as justificativas das mulheres por terem passado pela cirurgia cesariana não foram coin- cidente com a indicação médica registrada nos prontuários. Aproximadamente $75 \%$ das mulheres investigadas tinham expectativas de que o parto fosse normal, principalmente pela ideia da recuperação mais rápida e $25 \%$ tinham a expectativa da cesariana, justificada principalmente por terem passado por situação anterior de cesariana. No entanto, o parto normal, esperado por 165 mulheres, ocorreu em $66 \%$ dos casos.

Bortolotto (2006) avaliou as frequências de partos vaginais e cesáreas em mulheres com doenças cardíacas e destacou que a realização da cirurgia estava relacionada à idade gestacional no parto menor que 37 semanas, possíveis intercorrências obstétricas e fetais e à insuficiência cardíaca na mãe, mas principalmente à presença anterior de cesárea. A prática repetida de cesariana fortalece o falso dogma de que após a cesariana, o parto normal seria inviável, o que se trata de um equívoco segundo Belizán, Althabe, Barros e Alexander (2000) e Potter e cols. (2001) ou de um tema ainda polêmico entre os médicos (Oliveira \& cols., 2002).

Potter e cols. (2001) destacam que, em quatro capitais brasileiras, aproximadamente $76 \%$ das mulheres que frequentaram hospitais públicos e $70 \%$ das que frequentaram hospitais privados, referiram preferência pelo parto vaginal, contra $13 \%$ e $19 \%$ de usuárias de hospitais públicos ou privados, respectivamente, que preferiam a cesariana. Perpétuo e cols. (1998) comentam que das 400 gestantes que participaram de seu estudo em Belo Horizonte, mais de $70 \%$ se submeteram à cesariana, contra as suas próprias expectativas. Do mesmo modo, Faúndes e Perpétuo (2002) destacam que apesar de quase $80 \%$ das gestantes terem expectativa pelo parto normal, a cesariana foi realizada em $72 \%$ das pacientes particulares e em $31 \%$ das atendidas no SUS; $64 \%$ das gestantes se submeteram à cesariana por indicação médica equivocada, ou, no mínimo, duvidosa, pois segundo os autores apenas $18 \%$ das gestantes se submeteram à cesariana por necessidade.

A maior parte das mulheres brasileiras prefere e deseja o parto normal à cesariana, mas acaba se submetendo à cirurgia por indicação do médico, de acordo com vários autores (Barbosa \& cols., 2003; Faúndes \& Perpétuo, 2002; Potter \& cols., 2001; Silva \& Costa, 2002). Existe no Brasil a cultura de que as mulheres preferem a cesariana como opção de parto, no entanto, o parto normal ainda é prioridade na escolha da maioria delas, tanto entre as que utilizam o serviço de saúde público, quanto o privado (Barbosa \& cols., 2003; Carniel \& cols., 2007). 
Estudando mulheres que já haviam experimentado as duas formas de parto ou apenas a cesariana, Faúndes, Pádua, Osis, Cecatti e Sousa (2004) observaram que a maioria delas declarou preferir o parto vaginal à cesariana, especialmente entre aquelas que já haviam experimentado os dois tipos. Em geral, os médicos acreditavam que elas temiam o parto vaginal e estavam satisfeitas por terem sido submetidas à cesárea, o que não correspondia ao relato das mulheres que declararam preferir o parto vaginal porque consideravam-no o que provocaria menor dor/sofrimento, tendo vivido os dois tipos de parto. As mulheres que preferiam o parto cesariano, também por acreditar ser esse o parto de menor sofrimento, haviam passado somente por cesárea anterior e desconheciam a experiência do parto normal. $\mathrm{O}$ desencontro de informações sobre as expectativas, temores e escolhas de parto entre as gestantes e médicos pode tanto sugerir que há uma precária comunicação no processo de pré-natal entre gestantes e profissionais da saúde em geral, quanto explicitar representações a respeito do parto e nascimento relacionadas ao universo sociocultural de cada uma dessas mulheres.

Quando há a preferência pelo tipo de parto vaginal os argumentos das mulheres têm sido: a) crença de que a recuperação após o parto seria mais rápida; b) concepção que seria uma escolha melhor e mais saudável para a mãe e para o bebê; c) associação da concepção deste parto como algo mais relacionado à natureza e d) experiência positiva com parto vaginal anterior (Carniel \& cols., 2007; Faúndes \& cols., 2004; Oliveira \& cols., 2002; Perpétuo \& cols., 1998).

Paterson-Brown e Fisk (1997) enfatizam que, muitas vezes, a cesariana é realizada porque há uma escolha materna para esse tipo de parto. A escolha pela cesariana entre as gestantes, muitas vezes não é baseada em esclarecimentos corretos e parece haver influência de outras pessoas nos argumentos favoráveis e desfavoráveis em relação aos tipos de parto (Hotimsky \& cols., 2002; Silva \& Costa, 2002; Silveira $\&$ Santos, 2004), mas as reais motivações que direcionam as escolhas de gestantes sobre o tipo de parto ainda parecem obscuras. Além disso, há diferentes variáveis que interferem na escolha por determinado tipo de parto e nem sempre é possível que a escolha da gestante se realize, o que pode gerar mais angústia, frustração e ansiedade.

Quando há a preferência pela cesariana, os argumentos das mulheres têm sido: a) crença na diminuição de dor, no período pré-parto e no próprio parto, b) crença de que a anatomia e a fisiologia da vagina seriam preservadas, c) concepção de que seria possível garantir a saúde do(a) filho(a) por entenderem haver menor risco ao bebê do que no parto vaginal, d) concepção de que a cesariana é uma forma moderna de parto e, finalmente e) como um modo de garantir o direito de controle sobre seu próprio corpo (Barbosa \& cols., 2003; Carniel \& cols., 2007; Faúndes \& Cecatti, 1991; Oliveira \& cols., 2002; Perpétuo \& cols., 1998; Souza, 1994).

Pesquisas têm destacado a importância da decisão da gestante a respeito do tipo de parto a que vai ser submetida, após o recebimento de informações prévias sobre os custos e benefícios dos procedimentos (Belizán \& cols., 2000; Graham, Hundley, Hall, Gurney \& Milne, 1999; Hotimsky \& cols., 2002; Roberts, Tracy \& Peat, 2002). No entanto, pouco se tem oferecido às gestantes, em serviços de assistência pré-natal, sobre esses esclarecimentos (Hotimsky \& cols., 2002; Oliveira \& cols., 2002).

Pesquisando as orientações que as gestantes receberam antes do parto, Silveira e Santos (2004), concluíram que $71 \%$ das mulheres relataram não haver recebido orientação educativa para o parto durante o pré-natal. Entre as 195 mulheres que lembraram alguma orientação recebida, a taxa de cesariana foi de $36 \%$ e essas orientações incluíam características do parto vaginal e da cesariana, sinais de trabalho de parto, incentivo à cesariana ou ao parto normal.

A importância de existir assistência pré-natal adequada, como bem argumentam Oliveira e cols. (2002), é fundamental, uma vez que, a partir do oferecimento de informações precisas que auxiliem as gestantes a compreenderem melhor os riscos e os benefícios, os mitos e as crendices relacionados ao parto, bem como sobre a condição pessoal, é que as gestantes poderiam se sentir mais tranquilas e seguras em relação ao parto, seja ele qual for. Estudos têm alertado sobre a pertinência da assistência à gestante durante o parto e pósparto, fases em que as mulheres necessitam de acolhimento de profissionais da área da saúde e de familiares (Almeida, 2005; Bocardi, 2004; Bruggemann, Parpinelli \& Osis, 2005; Deslandes, 2005; Domingues, Santos \& Leal, 2004; Gomes \& Guedes, 2004; Granato, 2000; Hotimsky e cols., 2002). Profissionais da área de Psicologia podem contribuir sobremaneira junto às equipes de saúde que lidam com o atendimento de assistência às gestantes, parturientes ou puérperas (Bruggemann \& cols., 2005; Gomes \& Guedes, 2004).

Compreender a saúde como um fenômeno multideterminado e trabalhar com as diferentes variáveis relacionadas a ela, pode fazer uma grande diferença na 
atuação dos psicólogos na área da saúde (Kubo \& Botomé, 2001). Esses autores destacam que "o âmbito de atuação da Psicologia não se limita às interferências sobre variáveis de natureza apenas psicológica, mas abrange todas as relações possíveis entre as variáveis psicológicas e as demais que se relacionam com o fenômeno "saúde"” (p. 100). Como uma área interdisciplinar, profissionais da Psicologia da Saúde, como defendem esses autores, podem contribuir com equipes de agentes educativos, incentivando e realizando ações de esclarecimento e acolhimento às gestantes, minimizando as situações estressoras tanto no prénatal, quanto no nascimento e no puerpério.

Considerando a importância em verificar o relato de gestantes sobre suas concepções a respeito do parto cesariano ou normal, pretendeu-se investigar as preferências, temores e justificativas para o parto escolhido por elas, realizando este estudo que tem por objetivos: (a) investigar a preferência pelo tipo de parto entre as gestantes e os motivos da preferência; (b) investigar se a gestante teme, ou não, o momento do parto, quais suas justificativas e o que minimiza o temor.

\section{MÉTODO}

\section{Participantes}

Participaram do estudo quarenta gestantes primigestas, entre 14 e 42 anos de idade. Apenas uma gestante tinha a idade superior a 40 anos, sendo assim, a média de idade entre as participantes, excluindo-se essa última, foi de 21 anos. Todas frequentavam um Centro de Saúde Municipal, para realização de exames pré-natal, em uma cidade do interior paulista. O nível de escolaridade das gestantes variou entre não alfabetizadas e o ensino médio completo, sendo que mais da metade (63\%) não havia completado o ensino fundamental. No que se refere ao estado civil, $75 \%$ das gestantes eram casadas ou viviam com seus companheiros, $25 \%$ eram solteiras e $5 \%$ haviam se separado de seus parceiros.

\section{Materiais}

Utilizou-se para a coleta de dados um roteiro de entrevista semi-estruturado, elaborado pelos pesquisadores, que incluía questões relativas à: (a) dados só- cio-demográficos; (b) condições pessoais de nascimento - tipo de parto e história do mesmo; (c) temores quanto ao momento do parto; (d) preferência por tipo de parto e justificativas; (e) influências recebidas para a tomada de decisão em relação ao tipo de parto; e (f) condições que deixariam a gestante mais tranquila no momento do parto.

\section{Procedimento}

O projeto de pesquisa foi aprovado pelo Comitê de Ética em Pesquisa da instituição e as participantes assinaram o Termo de Consentimento Livre e Esclarecido, o que garantiu a participação voluntária.

A coleta de dados foi realizada nas dependências do próprio Centro de Saúde, em uma sala reservada, com privacidade adequada e livre de ruídos e interferências externas. As entrevistas ocorreram individualmente, antes de a gestante entrar no atendimento prénatal agendado para aquele dia. As entrevistas não foram gravadas, por solicitação do local que não permitia o uso de equipamentos de áudio e vídeo. Assim, as verbalizações das gestantes foram registradas por escrito, por um pesquisador treinado, e a duração média foi de 30 minutos. Todo o período da coleta de dados teve duração de três meses. É importante destacar que na situação da coleta de dados nenhuma gestante apresentava sintomas clínicos, físicos ou psicológicos atípicos e também não estavam em trabalho de parto iminente.

A análise dos dados foi qualitativa e quantitativa. As respostas foram quantificadas em termos de frequência, elaborando-se um sistema de categorias para analisar os motivos pelos quais as gestantes optavam por determinado tipo de parto e os motivos para temer, ou não, o procedimento. $\mathrm{O}$ sistema obedeceu aos critérios de inclusão de toda e qualquer resposta das gestantes, englobando apenas um tipo de resposta em cada categoria e descrevendo-as de forma objetiva e concisa, seguindo a análise de conteúdo proposta por Bardin (1977) e Gomes (1993). As tabelas a seguir apresentam os agrupamentos das categorias utilizados na análise de dados relativos às preferências de parto (Tabela 1) e a presença ou não de temor ao parto (Tabela 2). Os dados foram quantificados por categorias de análise e as porcentagens de frequência calculadas. 
TABELA 1

Agrupamentos dos Relatos das Gestantes Distribuídos em Categorias de Análise Relativas à Preferência do Tipo de Parto

\begin{tabular}{|c|c|c|}
\hline Categorias de análise & & Agrupamentos dos relatos por categorias \\
\hline \multirow{5}{*}{$\begin{array}{l}\text { Pela preferência do } \\
\text { parto normal }\end{array}$} & Rápida recuperação & $\begin{array}{l}\text { "o pós-operatório do parto normal é mais fácil"; "a dor está limitada ao momento do parto"; } \\
\text { "logo em seguida é possível andar ou trabalhar"; "o sofrimento na cesariana é maior", "a } \\
\text { recuperação é mais lenta e dolorosa" }\end{array}$ \\
\hline & $\begin{array}{l}\text { Melhor para a mãe e para } 0 \\
\text { bebê }\end{array}$ & $\begin{array}{l}\text { "é mais saudável devido à ausência da anestesia", "ausência de incisão pélvica e/ou porque } \\
\text { o leite é mais abundante" }\end{array}$ \\
\hline & Condição financeira & "sem recursos para pagar pela cesariana, apesar de que prefere o normal" \\
\hline & Ausência de cicatriz & "não quer ter um corte no abdômen" \\
\hline & $\begin{array}{l}\text { Conselhos do médico ou de } \\
\text { outras pessoas }\end{array}$ & "o médico ou conhecido disse que o parto normal é melhor" \\
\hline \multirow{2}{*}{$\begin{array}{l}\text { Pela preferência do } \\
\text { parto cesariana }\end{array}$} & Menor sofrimento & $\begin{array}{l}\text { "não sentir as dores do parto", "ter acesso a anestesia" e/ou "o trabalho do parto é mais } \\
\text { rápido" }\end{array}$ \\
\hline & Indicação médica & $\begin{array}{l}\text { "sugestão do médico pela cesariana" e ou "aproveitaria a oportunidade para realização de } \\
\text { procedimento cirúrgico adicional" }\end{array}$ \\
\hline
\end{tabular}

TABELA 2

Agrupamentos dos Relatos das Gestantes Distribuídos em Categorias de Análise Relativas à Presença ou Ausência de Temor Diante do Parto

\begin{tabular}{|c|c|c|}
\hline Categorias de análise & & Agrupamentos dos relatos por categorias \\
\hline \multirow{7}{*}{$\begin{array}{l}\text { Pela presença de } \\
\text { receio/temor que } \\
\text { antecede o parto }\end{array}$} & Sentir dor/sofrer & $\begin{array}{l}\text { "medo de sentir dor e/ou sofrer com o parto devido aos efeitos da anestesia ou do pós- } \\
\text { operatório" }\end{array}$ \\
\hline & $\begin{array}{l}\text { Receio de acontecer algo } \\
\text { com elas e/ou com o bebê }\end{array}$ & $\begin{array}{l}\text { "medo de morrer por complicações do parto" ou "receio do bebê nascer com problemas ou } \\
\text { vir a óbito" }\end{array}$ \\
\hline & Desconhecido & $\begin{array}{l}\text { "receio da hospitalização ou do parto, porque nunca viveram essa situação antes", e ou } \\
\text { "medo da realidade diante de tantas informações desencontradas" }\end{array}$ \\
\hline & $\begin{array}{l}\text { Experiência de outras } \\
\text { mulheres }\end{array}$ & $\begin{array}{l}\text { "conhecimento a partir de outra pessoa que já havia passado por determinado parto e que } \\
\text { este havia sido muito difícil" }\end{array}$ \\
\hline & $\begin{array}{l}\text { Ter que fazer o parto não } \\
\text { desejado }\end{array}$ & "receio de se submeter a um tipo de parto diferente daquele que gostaria" \\
\hline & Experiência prévia & "medo porque já tinham passado por outras cirurgias e internações" \\
\hline & Não soube explicar & "quando as gestantes referiam algum receio, mas não conseguiam expressar a razão" \\
\hline \multirow{4}{*}{$\begin{array}{l}\text { Pela ausência de } \\
\text { receio/temor que } \\
\text { antecede o parto }\end{array}$} & Processo natural & $\begin{array}{l}\text { "ideia de que o parto normal é um processo natural e que grande parte das mulheres passa } \\
\text { por isso" }\end{array}$ \\
\hline & Ver o bebê & "a vontade de ver o bebê está acima de qualquer possível dificuldade" \\
\hline & Experiência prévia & "ausência de medo porque já tinham passado por cirurgias e internações" \\
\hline & Não soube explicar & $\begin{array}{l}\text { "quando as gestantes referiam não ter nenhum medo, mas não conseguiam expressar a } \\
\text { razão" }\end{array}$ \\
\hline
\end{tabular}

\section{RESULTADOS}

\section{Preferência pelo tipo de parto entre as gestantes e os motivos da preferência}

Observou-se que das 40 gestantes entrevistadas, $75 \%$ relataram preferir o parto normal, $15 \%$ a cesariana e $10 \%$ ainda não tinham escolhido o tipo de parto na ocasião da entrevista. Vale lembrar que a gestante poderia responder a opção do parto como uma escolha, independentemente de haver ou não a indicação verbal por parte do médico para um determinado tipo de parto.

A principal justificativa pela opção do parto normal está representada na categoria que agrupa relatos 
sobre a rápida recuperação $(62 \%)$, seguida pela consideração de ser um tipo de parto mais saudável para a mãe e para o bebê (24\%). A outra categoria, denominada Outros $(14 \%)$, reúne respostas relacionadas à condição financeira, ausência de cicatriz e conselhos de outras pessoas. A preferência pela cesariana é representada, na maioria dos casos, pela categoria menor sofrimento $(80 \%)$, seguido da categoria indicação médica $(20 \%)$.

Curiosamente, as justificativas mais representativas para a escolha do parto normal e a escolha pelo parto cesariano (rápida recuperação e menor sofrimento, respectivamente), enfocam crenças positivas, isto é, justificativas que favorecem aspectos relacionados ao bem estar pessoal. Ao escolherem o parto normal, as gestantes ressaltam os aspectos favoráveis como pós-operatório mais fácil; dor limitada ao parto; rápida recuperação para locomoção e trabalho ("logo em seguida é possível andar ou trabalhar") e ao escolherem o parto cesariano o mesmo padrão parece ser observado: não sentir as dores do parto, trabalho do parto mais rápido. Pode-se, então, sugerir que as justificativas pela escolha do parto são motivadas pela vontade de afastar ou diminuir sentimentos de dor e sofrimento atribuído às situações de parto.

As justificativas das participantes para a escolha do tipo de parto pode ser resumida da seguinte forma: Escolhas para o Parto Normal: rápida recuperação da mãe (62\%); melhor para mãe e para o bebê (24\%); e outros (14\%) e escolhas para o Parto Cesariano: menor sofrimento para a mãe e bebê $(80 \%)$ e por corresponder a indicação médica $(20 \%)$.
As gestantes também relataram sobre as pessoas que influenciaram a sua opção pelo parto normal ou cirúrgico, destacando, em ambos os grupos, pessoas de seu convívio cotidiano com quem mantinham relações de afeto e confiança, seja por parentesco ou amizade. A opinião favorável ou não de outras mulheres sobre determinado parto pareceu importante fonte de influência. Das trinta gestantes que escolheram o parto normal, 24 relataram que suas mães, outras mulheres da família ou amigas/conhecidas foram as principais influências sobre a escolha do parto. As mesmas influências foram relatadas por oito das 11 gestantes que escolheram o parto cesariano. Ou seja, nos dois casos de escolhas de partos, as fontes de influências mantiveram-se as mesmas: relatos de outras mulheres e opinião de familiares. As outras influências relatadas pelas demais gestantes incluíram o fato de receberem informações e/ou indicações do médico ou por terem tido contato com narrativas midiáticas ("assistir a filmes").

\section{Presença ou ausência de medo/temor entre as gestantes diante do parto, suas justificativas e 0 que minimiza o temor}

Parece comum entre as gestantes o relato de sentimentos de medo, receio e temor diante da situação do parto. Das 40 gestantes, $29(72,5 \%)$ assumiram esse sentimento de temor, e desta parcela, 21 tinham optado pelo parto normal e oito pela cesariana. A Figura 1 mostra os motivos de medo, no momento do parto, relatados pelas gestantes.

\section{Justificativas para o medo do parto}

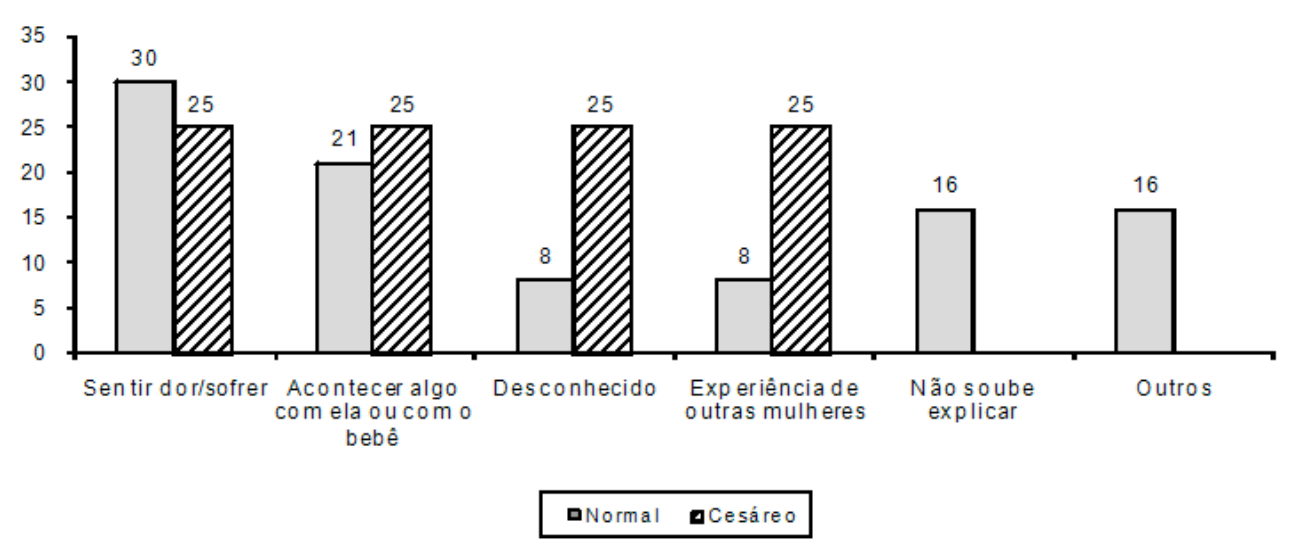

Figura 1. Agrupamentos em categorias sobre as justificativas das gestantes para o sentimento de medo/temor diante do parto. Eixo vertical indica a porcentagem de respostas e 0 eixo horizontal os agrupamentos de respostas. 
Entre as gestantes que optaram pelo parto normal, as principais razões para os sentimentos de receio/ temor referem-se às categorias sentir dor e sofrer $(30 \%)$ ou acontecer algo com elas e/ou com o bebê $(25 \%)$ e $16 \%$ das gestantes não souberam explicar o temor. As gestantes que optaram pela cesariana apresentaram justificativas sobre o medo/temor distribuídas, de modo semelhante, entre as categorias sentir dor/sofrer $(25 \%)$, receio de que aconteça algo com elas e/ou com o bebê (25\%), receio de enfrentar o desconhecido $(25 \%)$ e experiência negativa vivenciada por outras mulheres $(25 \%)$. Aqui também é possível observar que o que gera ansiedade e medo envolve tanto o sentimento de dor física quanto o sentimento de cuidado e preocupação com o bem estar pessoal e do bebê, seja entre as gestantes que escolheram o parto normal ou entre as que escolheram a cesariana.

Independentemente do tipo de parto escolhido, as 17 gestantes que não relataram sentimentos de temor pelo momento do parto apresentaram os seguintes argumentos: percebem o parto como um processo natural $(36,5 \%)$, a vontade de ver/conhecer o bebê está acima de tudo (18\%) e já foram expostas a procedimentos cirúrgicos anteriores (9\%). Outras gestantes $(36,5 \%)$ não souberam explicitar o motivo da ausência de medo. Evidentemente, relatar o medo envolve a identificação de eventos estressores, o que pode ser o caso dessas gestantes que não descreveram essas ocorrências prévias. A justificativa de ausência de medo por já terem vivenciado a procedimentos cirúrgicos anteriores, seria o caso mais provável de relatos baseados na exposição às contingências anteriores reforçadoras, o que ocorreu em menor índice nesta amostra sobre o relato de ausência de medo, apenas $9 \%$ das gestantes.

Finalmente, a análise das verbalizações das gestantes agrupa as diferentes condições que envolveriam situações de bem estar e tranquilidade no momento do parto: a) estarem acompanhadas da presença de alguma pessoa de seu convívio (61\%), como o marido, a mãe e outras familiares/amigas e também o médico que acompanhou o pré-natal; b) receberem informações sobre a saúde pessoal e sobre o sucesso do parto $(31 \%)$. As demais gestantes $(8 \%)$ não souberam dizer o que ou quem as ajudaria a minimizar o temor do parto.

\section{DISCUSSÃO}

A preferência quanto ao tipo de parto, a partir do relato das gestantes neste estudo, foi pelo parto vaginal (normal), o que corrobora com dados sobre a pre- ferência do parto encontrada na literatura (Barbosa \& cols., 2003; Carniel \& cols., 2007; Faundes \& Perpétuo, 2002; Hopkins, 2000; Miranda, 2003; Oliveira \& cols., 2002; Perpétuo \& cols., 1998; Silva \& Costa, 2002; Sociedade Civil de Bem-Estar Familiar no Brasil, 1997) e indica que a concepção do parto normal como algo desejável ainda faz parte das representações culturais sobre o nascimento. Isso indica, ratificando autores como Barbosa e cols. (2003), Hotimsky e cols. (2002) e Oliveira e cols. (2002) que, apesar dos elevados índices de cesarianas no país, as expectativas das mulheres ainda são pelo parto normal, especialmente entre as primigestas.

As justificativas para preferência pelo parto normal também foram condizentes com as encontradas na literatura (Carniel \& cols., 2007; Hotimsky \& cols., 2002; Oliveira \& cols., 2002; Perpétuo \& cols., 1998; Potter \& cols., 2001; Quadros, 2000; Souza, 1994), reforçando a ideia de que aspectos positivos para o bem estar materno e do(a) filho(a), isto é, a "rápida recuperação", quando comparado ao parto cesariana, e o julgamento de se tratar da melhor opção para a mãe e o bebê.

Todavia, é preciso considerar que entre as que escolheram o parto cesariano, as crenças na recuperação positiva também apareceram. Entre as mães que optaram pelo parto normal havia o argumento de que a recuperação seria mais favorável para a mãe e o contato com o seu bebê facilitado, mas entre as que optaram pela cesariana havia também argumentos de que seria um parto no qual se sofre proporcionalmente menos, se comparado ao normal; esta foi uma tendência encontrada também entre as gestantes que preferiram a cesariana, no estudo de Perpétuo e cols. (1998). Em ambas as escolhas de parto, as gestantes revelaram crenças de afastamento de dor/evitar o sofrimento momentâneo ou pós-parto, ratificado por Oliveira e cols. (2002), que também observaram as mesmas justificativas entre as mulheres que desejavam tanto o parto normal quanto a cesariana: "parto mais rápido" e "menor dor e sofrimento".

Das gestantes que disseram que não tinham medo do parto, seja o vaginal ou a cesariana, a maior parte explicou que considerava o parto um processo natural ou que o nascimento do bebê superava a ansiedade antecipatória pela exposição ao procedimento ou, ainda, que já haviam sido expostas a outros procedimentos cirúrgicos e conheciam tal contexto.

Entre as participantes deste estudo que optaram pelo parto normal, os receios mais expressivos estavam relacionados a: medo de sentir dor, medo de so- 
frer ou medo de ocorrer algum imprevisto com elas e/ou com o bebê. Entre as gestantes que optaram pela cesariana, a maior parte apresentou medo de enfrentar a situação, seja pela expectativa de dor ou de sofrimento, pelo conhecimento da experiência adversa vivenciada por outras mulheres, receio de que algo pudesse acontecer com elas ou com o bebê ou, ainda, de enfrentarem uma situação desconhecida. Hotimsky e cols. (2002) também encontraram essas justificativas entre as gestantes que temiam a cesariana e desejavam o parto normal: "recuperação pós-parto mais rápida" $\mathrm{e}$ "medo da cirurgia e de possíveis sequelas"; entre as que desejariam a cesariana, não havia temor ao parto normal, mas justificativas da necessidade da cesariana eram priorizadas no discurso das gestantes: "idade avançada", "cesariana anterior", "associação da laqueadura".

Tais receios, em geral, evidenciam a pertinência das argumentações de alguns autores que defendem a necessidade da humanização do parto, estendida a toda população, como uma condição fundamental para a minimização dos receios das gestantes em optar pelo parto (Barros, Vaughan, Victora \& Huttly, 1991; Diniz, 2005; Graham \& cols., 1999; Patterson-Brown \& Fisk, 1997; Rezende, 2002; Roberts \& cols., 2002). Destaca-se que a mesma preocupação deveria ocorrer para todas as gestantes, também àquelas que realizam o parto cirúrgico, seja por opção pessoal ou indicação médica. O profissional da psicologia da saúde deveria estar atento a essas questões e atuar junto com outros agentes no sentido de esclarecer e acolher a ansiedade de primigestas em período de definição de parto.

As gestantes, em geral, carecem de orientações e esclarecimentos sobre o trabalho de parto, que deveriam fazer parte de uma assistência integral à gestante (Hotimsky \& cols., 2002; Silveira \& Santos, 2004), o que reforça a ideia de que é importante discutir a necessidade de esclarecer às gestantes sobre a exposição a procedimentos médicos sob contingências ambientais de tratamento que garantam humanização e atendimento às necessidades psicossociais dos pacientes.

Os relatos sobre as explicações que geravam sentimentos de temor e medo diante dos procedimentos de parto foram mais evidentes quando as gestantes identificaram esse sentimento de medo, seja diante do parto normal ou cesariano. Porém, dentre as gestantes que disseram que "não tinham medo do parto", nem todas foram capazes de explicar seus motivos. Isso sugere a necessidade de investigações mais detalhadas para uma melhor compreensão do aspecto do medo, uma vez que pela própria definição essa sensação exige a indicação do objeto ou evento potencialmente desencadeante. No entanto, pode-se levantar a hipótese de que aquelas que alegaram não sentir medo e não explicaram o porquê foram as gestantes que não apresentaram alguma condição de ansiedade antecipatória. Do mesmo modo, deve haver situações, como exposição anterior a experiências pessoais ou a relatos de terceiros, que desencadeiam crenças e concepções sobre o parto ideal ou menos sofrível. Vale à pena destacar que Faúndes e cols. (2004) observaram a preferência pelo parto normal entre as gestantes que tinham tido a experiência desse parto em gestações anteriores.

As participantes afirmaram que foram influenciadas principalmente por pessoas próximas ou familiares para optar pelo parto que fizeram, ratificando dados de Hotimsky e cols. (2002). Se a maioria das mulheres grávidas pela primeira vez optou pelo parto normal, provavelmente, são mulheres que foram expostas a comentários positivos sobre este tipo de parto por pessoas conhecidas e não por terem recebido informações das próprias instituições de saúde em que realizam o pré-natal.

As gestantes indicaram que seria tranquilizadora a presença de um acompanhante escolhido por elas no momento do parto, o que, segundo Almeida (2005) e Bruggemann e cols. (2005), representaria um suporte emocional importante para a parturiente. Há hospitais que têm adotado a prática de permitir a presença do marido e eventualmente outro familiar, o que exige do hospital uma infraestrutura física e recursos humanos adequados para receber e manter acompanhantes em contexto de sala de parto e centro cirúrgico (Bruggemann \& cols., 2005). O psicólogo, mais uma vez, seria um profissional que poderia alertar para essa demanda e buscar alternativas para minimizar o sofrimento da gestante e de seus familiares.

Além disso, as gestantes também se referiram à importância da presença do médico que as acompanhou durante o pré-natal na hora do parto, reforçando preocupações de Caprara e Rodrigues (2004) sobre a importância da relação médico-paciente. Reitere-se a relevância do estabelecimento de vínculos positivos, de confiança e segurança entre pacientes e profissionais de saúde e a importância de humanizar as maternidades e os atendimentos às gestantes. Muitos autores têm defendido a importância da dedicação de uma equipe interdisciplinar na assistência pré-natal e a concepção de humanização do parto (Deslandes, 2005; Domingues \& cols., 2004; Moreira, 2006; Silveira \& Santos, 2004; Serruya \& cols., 2004), inclusive ressaltando o papel da psicologia nessa assistência (Gomes \& Guedes, 2004; Granato, 2000). 


\section{CONSIDERAÇÕES FINAIS}

Os resultados deste estudo e os dados da literatura consultada permitem levantar a hipótese de que as gestantes tendem a apresentar razões semelhantes para a opção pelo parto normal ou pela cesariana no sentido de afastar a dor e sofrimento, sobretudo a partir de representações sociais sobre parto e nascimento que se expressam, resultante talvez da falta de informação ou crenças pessoais, em temores e argumentos diversos. Nesse sentido, seria importante que os profissionais de saúde se engajassem mais sistematicamente em campanhas de esclarecimentos acerca das vantagens e desvantagens dos diversos tipos de parto, diferenciando-os em função das necessidades e condições clínicas e psicossociais de cada gestante, de modo que essas representações sobre os tipos de parto fossem contrastadas por informações e esclarecimentos adequados.

A identificação dos temores e das crenças de gestantes diante do parto poderia contribuir no esforço de diferentes profissionais da saúde em ajudar as mulheres a receber esclarecimentos necessários sobre os riscos e benefícios em diferentes partos, ou mesmo a lhes oferecer conforto, garantindo a presença de pessoas conhecidas ou a lhes proporcionar a diminuição da ansiedade diante do medo de sentir dor ou medo do "desconhecido". Assim, disponibilizar assistência prénatal sistemática e adequada, que inclua informações claras e atualizadas às gestantes, bem como o oferecimento de cursos preparatórios, poderia assegurar sentimentos de segurança e confiança às gestantes.

Além disso, estudos sobre os mecanismos psicológicos e subjetivos que atuam nos comportamentos de pessoas em situações de hospitalização, geralmente estressantes e desconhecidas, poderiam contribuir para o levantamento de ocorrências que desencadeassem o aumento de ansiedade das gestantes diante do parto e nascimento, colaborando com estratégias preventivas. Por um lado, há ocorrência de cesarianas em função da comodidade do médico e/ou pela necessidade de garantir o bem estar materno e fetal; por outro lado há concepções sobre o nascimento que influenciam as expectativas de mulheres a respeito do parto desejado. Evidentemente que a opção para o nascimento não deve levar em conta apenas o desejo da gestante, considerando possíveis e necessárias indicações médicas específicas de cada caso. Novos estudos, considerando um número mais expressivo de gestantes em diferentes contextos ou ainda que investiguem o efeito da preparação pré-natal sobre o repertório de comporta- mentos de enfrentamento da gestante, tanto sobre os procedimentos cirúrgicos que envolvem o parto, quanto sobre a influência desses procedimentos para a saúde, poderiam contribuir na elucidação dessa temática tão importante para vários profissionais e pesquisadores da área da saúde, como é o caso da Psicologia.

\section{REFERÊNCIAS}

Almeida, M. S. (2005). Assistência de enfermagem à mulher no período puerperal: Uma análise das necessidades como subsidios para a construção de indicadores de gênero. Tese de doutorado não-publicada, Universidade de São Paulo, Ribeirão Preto.

Barbosa, G. P., Giffin, K., Ângulo-Tuesta, A., Gama, A. S., Chor, D., D’Orsi, E. \& Reis, A. C. G. V. (2003). Parto cesáreo: Quem o deseja? Em quais circunstâncias?. Cadernos de Saúde Pública, 19(6), 1611-1620.

Bardin, L. (1977). Análise de conteúdo (L. A. Reto \& A. Pinheiro, Trad.). Rio de Janeiro: Edições 70.

Barros, F. C., Vaughan J. P., Victora C. G., \& Huttly, S. H. (1991). Epidemic of caesarean sections in Brazil. The Lancet, 338, 167-169.

Belizán, J. M., Althabe, F., Barros, F., \& Alexander, S. (2000). Caesarean section controversy: Authors' reply. British Medical Journal, 320, 1072-1073.

Bocardi, M. I. B. (2004). Assistência pré-natal na adolescência: Concepções das adolescentes e dos profissionais da saúde. Tese de doutorado não-publicada, Universidade de São Paulo, Ribeirão Preto.

Bortolotto, M. R. F. L. (2006). Estudo dos fatores relacionados à determinação da via do parto em gestantes portadoras de cardiopatias. Tese de doutorado não-publicada, Universidade de São Paulo, São Paulo.

Bruggemenn, O. M., Parpinelli, M. A., \& Osis, M. J. D. (2005). Evidências sobre o suporte durante o trabalho de parto: Uma revisão da literatura. Cadernos de Saúde Pública, 21(5), 13161327.

Caprara, A., Rodrigues, J. (2004). A relação assimétrica médicopaciente: Repensando o vínculo terapêutico. Ciência \& Saúde Coletiva, 9(1), 139-146.

Carniel, E. F., Zanolli, M. L. \& Morcillo, A. M. (2007). Fatores de risco para indicação do parto cesáreo em Campinas (SP). Revista Brasileira de Ginecologia e Obstetrícia, 29(1), 34-40.

Dias, M. A. B., \& Deslandes, S. F. (2004). Cesarianas: Percepção de risco e sua indicação pelo obstetra em uma maternidade pública no município do Rio de Janeiro. Cadernos de Saúde Pública, 20(1), 109-116.

Diniz, C. S. G. (2005). Humanização da assistência ao parto no Brasil: Os muitos sentidos de um movimento. Ciência \& Saúde Coletiva, 10(3), 627-637. 
Diniz, C. S. G \& Duarte, A. C. (2004). Parto normal ou cesárea? $O$ que toda mulher deve saber (e todo homem também). São Paulo: Unesp.

Deslandes, S. F. (2005). A ótica de gestores sobre a humanização da assistência nas maternidades municipais do Rio de Janeiro. Ciência e Saúde Coletiva, 10 (3), 615-626.

Domingues, R. M. S. M., Santos, E. M., \& Leal, M. C. (2004). Aspectos da satisfação das mulheres com a assistência ao parto: Contribuição para o debate. Cadernos de Saúde Pública, 20(Supl. 1), S52-S62.

Faisal-Cury, A., \& Menezes, P. R. (2006). Fatores associados à preferência por cesareana. Revista de Saúde Pública, 40(2), 226-232.

Faúndes, A., \& Cecatti. J. G. (1991). A operação cesárea no Brasil: Incidência, tendências, causas, consequências e propostas de ação. Cadernos de Saúde Pública, 7, 150-173.

Faúndes, A. \& Perpétuo, H. O. (2002). Cesárea por conveniência e a ética médica. Ser Médico, 5, 32-34.

Faúndes, A., Pádua, K. S., Osis, M. J. D., Cecatti, J. G., \& Sousa, M. H. (2004). Opinião de mulheres e médicos brasileiros sobre a preferência pela via de parto. Revista de Saúde Pública, 38(4), 488-494.

Gama, E. (2007). O retorno ao parto normal. Portal da Família. Retirado em 5 de outubro de 2007, de http://www.portalda familia.org/artigos/artigo086.shtml.

Gomes, R. (1993). Análise e interpretação de dados de pesquisa qualitativa. Em M. C. S. Minayo (Org.), Pesquisa social: Teoria, método e criatividade (pp. 79-108). Petrópolis: Vozes.

Gomes, L. A., \& Guedes, C. R. (2004). A preparação para o parto como prevenção primária com grupos de sala de espera: A experiência de trabalho interprofissional. Psicologia Hospitalar, 2(2), 1-7.

Graham, W. J., Hundley, V., Mccheine, A. L., Hall, M. H., Gurney, E., \& Milne, J. (1999). An investigation of women's involvement in the decision to deliver by caesarean section. British Journal of Obstetrics and Gynecology, 106, 213-220.

Granato, T. M. M. (2000). Encontros terapêuticos com gestantes à luz da preocupação materna primária. Dissertação de mestrado não-publicada, Universidade de São Paulo.

Halvorsen, L., Nerum, H., Oian, P., \& Sorlie, T. (2008). Is there an association between psychological stress and request for caesarian section? Tidsskr Nor Laegeforen, 128(12), 13881391.

Hopkins, K. (2000). Are Brazilian women really choosing to deliver by caesarean? Social Science \& Medicine, 51(110), 725-740.

Hotimsky, S. N., Rattner, D., Venancio, S. I., Bógus, C. M., \& Miranda, M. M. (2002). O parto como eu vejo... Ou como eu desejo? Expectativas de gestantes, usuárias do SUS, acerca do parto e da assistência obstétrica. Cadernos de Saúde Pública, 18(5), 1303-1311.
Kubo, O. M., \& Botomé, S. P. (2001). Formação e atuação do psicólogo para o tratamento em saúde e em organizações de atendimento à saúde. Interação em Psicologia, 5, 93-122.

Martin-Costa, S., Ramos, G. L. (2005). A questão das cesarianas [Editorial]. Revista Brasileira de Ginecologia e Obstetrícia, 27(10), 571-574.

Miranda L. (2003, 22 de junho). Grávidas querem parto normal. Médicos, cesárea. O Estado de São Paulo, p. A14.

Novas ações para reduzir as cesarianas na saúde suplementar. (2009, janeiro/fevereiro). Jornal Unidas - União Nacional das Instituições de Autogestão em Saúde, n. 43, 4-5.

Oliveira, S. M. J. V., Riesco, M. L. G., Miya, C. F. R., \& Vidotto, P. (2002). Tipo de parto: Expectativas das mulheres. Revista Latinoamericana de Enfermagem, 10(5), 667-674.

Paterson-Brown, S., \& Fisk, N. M. (1997). Caesarean section: Every woman's right to choose? Current Opinion in Obstetrics and Gynecology, 9(6), 351-355.

Perpétuo, I. H. O., Bessa, G. H., \& Fonseca, M. C. (1998). Parto cesáreo: Uma análise da perspectiva das mulheres de Belo Horizonte. Em Associação Brasileira de Estudos Populacionais (Org.), Anais do XI Encontro Nacional de Estudos Populacionais da ABEP (pp. 95-119). São Paulo: ABEP.

Potter, J. E., Berquó, E., Perpétuo, I. H. O., Leal, O. F., Hopkins, K., \& Souza, M. R. (2001). Unwanted caesarean sections among public and private patients in Brazil: Prospective study. British Medical Journal, 323, 1155-1158.

Quadros, L. G. (2000). Caesarean section controversy: Brazilian obstetricians are pressured to perform caesarean sections. British Medical Journal, 320(7241), 1073.

Rezende J. (2002). A falácia do parto humanizado. Femina, 30(7), 481-484.

Roberts, C. L., Tracy, S., \& Peat, B. (2000). Rates for obstetric intervention among private and public patients in Australia: Population based descriptive study. British Medical Journal, 321, 137-141.

Serruya, S. J., Lago, T. D., \& Cecatti, J. G. (2004). O panorama da atenção pré-natal no Brasil e o Programa de Humanização do Pré-natal e Nascimento. Revista Brasileira de Saúde Materno Infantil, 4(3), 269-279.

Silva, F. A. M., \& Costa, N. D. L. (2002). Estudo multicêntrico sobre saúde reprodutiva no Brasil: Alguns resultados da pesquisa no Rio Grande do Norte. Em Associação Brasileira de Estudos Populacionais (Org.), Anais do XIII Encontro Nacional de Estudos Populacionais da ABEP (pp. 1-25). Ouro Preto: ABEP.

Silveira, D. S., \& Santos, I. S. (2004). Fatores associados à cesariana entre mulheres de baixa renda em Pelotas, Rio Grande do Sul, Brasil. Cadernos de Saúde Pública, 20(2), 231-241.

Sociedade Civil de Bem-Estar Familiar no Brasil (1997). Pesquisa nacional sobre demografia e saúde. Rio de Janeiro: BENFAM/Macro International. 
Souza M. C. (1994). C-sections as ideal births: The cultural constructions of beneficence and patients' rights in Brazil. Cambridge Quarterly of Healthcare Ethic, 3(3), 358-366.

Yazlle, M. E. H. D., Rocha, J. S. Y., Mendes, M. C., Patta, M. C., Marcolin, A. C., \& Azevedo, G. D. (2001). Incidência de cesá- reas segundo fonte de financiamento da assistência ao parto. Revista de Saúde Pública, 35(2), 202-206.
Recebido: $28 / 11 / 2007$

Última revisão: 02/05/2009

Aceite final: 04/05/2009

\section{Nota:}

1 Agradecemos a Helena Cuelhar R. Martins pela colaboração durante a coleta de dados, e ao Prof. Dr. Áderson Costa Júnior pela valiosa contribuição na revisão do manuscrito.

\section{Sobre as autoras:}

Lígia Ebner Melchiori: Psicóloga. Doutora em Psicologia pela USP/Ribeirão Preto/SP; Pós-Doutoranda em Desenvolvimento Familiar, pela UnB/Brasília/DF.

Ana Cláudia Bortolozzi Maia: Psicóloga. Doutora em Educação pela UNESP/Marília/SP.

Rita Nathalia Bredariolli: Psicóloga pela UNESP/Bauru/SP.

Raquel Ishikawa Hory: Psicóloga. Mestre em Psicologia do Desenvolvimento e da Aprendizagem, pela UNESP/Bauru/SP.

Endereço para correspondência: Departamento de Psicologia/Faculdade de Ciências - Av. Eng. Luiz Edmundo Carrijo Coube, 14-01 Bairro Vargem Limpa - 17033-360 Bauru/SP - Endereço eletrônico: 1melch@fc.unesp.br, aclaudia@fc.unesp.br 